\title{
Multidisciplinary Approach to Tumor Pathologies: State of the Art of a Single Center
}

Anania $G^{1}$, Fabbri $N^{1}$, Marino $S^{1}$, Resta $G^{1}$, Giaccari $S^{1}$, Tamburini $N^{1}$, Fiorica $F^{2}$, Cavallesco $G^{1}$

'Department of Morphology, Experimental Medicine and Surgery, Section of General and Thoracic Surgery, University of Ferrara, Ferrara, Italy. ${ }^{2}$ Department of Radiation Oncology, University Hospital Ferrara, Ferrara, Italy.

Article Info

\section{Article Notes}

Received: May 23, 2018

Accepted: June 18, 2018

\section{*Correspondence:}

Dr. Fabbri N, Department of Morphology, Experimental Medicine and Surgery, Section of General and Thoracic Surgery, University of Ferrara, Ferrara, Italy;

Email: nicolo.fabbri@student.unife.it

(c) 2018 Fabbri N. This article is distributed under the terms of the Creative Commons Attribution 4.0 International License.

\section{ABSTRACT}

Aim: The figure of cancer patient has changed. Some reasons are the increase of the average population and higher incidence of diseases related to old age (cardiovascular diseases, diabetes, etc.), but also diseases such as obesity are increasing in industrialized countries. For these reasons is necessary to choose an adequate and personalized treatment, taking into consideration the different risk of mortality and morbidity in therapeutic choices through a greater interaction between medical specialists.

Experiment: we conducted a retrospective study that took into consideration all the patients who underwent surgery of rectosigmoid junction and rectum in our hospital before and after the institution of a multidisciplinary tumour board (MTB) from January 2007 to April 2017. Furthermore, a recent retrospective single-centre analysis was performed in the same hospital by Tamburini et al. on consecutive patients who underwent surgery for non-small cell lung cancer (NSCLC) between January 2008 and December 2015.

Results: the advantage brought by the multidisciplinary evaluation teams are confirmed for the presence of benefits in terms of reduction of morbidity and improvement of the outcome of MTB patient group.

Conclusions: we find that more study depth of neoplastic disease as well as of each individual patient leads to more accurate staging and to a weighted therapy based on the needs of the individual. According to our experience, multidisciplinary tumour board should be present in all hospitals where cancer diseases are treated.

\section{Discussion}

Colorectal cancer is the third most prevalent cancer in the world and is the fourth leading cause of death in men $(7.6 \%)$ and the third in women $(8.6 \%)$. In the recent past, patients were discussed in occasional meetings where different specialists participated in the definition of the treatment strategy.

In Italy attempts are made to encode a standardized path for patients suffering from cancer of the colon and rectum. The growing number of studies in favor of multidisciplinary approaches supports evidence-based medicine ${ }^{1}$.

A multidisciplinary tumour board (MTB) brings together all teams involved in patient's care, including physicians (oncologists, radiologists, anesthetists, surgeons, pulmonologists and pathologists), nurses, social workers, dieticians, physiotherapists and occupational therapists. The MTB members share their expertise, professional perspective and knowledge, and conferences 
are designed to enhance patient management and outcomes.

It has numerous advantages, such as the guarantee of a correct therapeutic decision for the precise neoplasm of the patient. Moreover, every participant is better protected from errors caused by the opinion of the individual. Importantly, the multidisciplinary discussion is a moment of growth for the joining specialists.

This methodology was introduced with the intent of providing more data for each patient, thus enabling better understanding of both primitive and potential secondary pathologies.

Our Unit of General and Thoracic Surgery joined the multidisciplinary program for cancer of the colon and rectum in S. Anna Hospital of Ferrara in 2012. We conducted a retrospective study that took into consideration all patients who underwent surgery of the rectosigmoid junction and rectum from January 2007 to April 2017.

The 96 patients included in the analysis were divided in two groups based on whether their preoperative care was coordinated by the multidisciplinary tumor board (MTB) or not.

The pre-MTB group included 45 patients operated between February 2007 and December 2011; the MTB group (51 patients) were treated between January 2012 and April 2017.

There were no significant differences in the average age of the patients before and after the multidisciplinary approach between the two groups.

The introduction of MTB in our clinical practice provided significant differences in terms of a more accurate preoperative patient's evaluation.

Some of the major differences between the two patient groups are:

- Number of preoperative chest and abdominal computed tomography scanning (CT) scan performed $(82,4 \%$ in MTB group, only $24,4 \%$ in preMTB group),

- Number of preoperative colonoscopy performed $(78,4 \%$ in MTB group, only $57,7 \%$ in pre-MTB group) ${ }^{1}$.

Computed tomography provided an opportunity to assess the stage of rectal cancer either locally or remotely, with an accuracy ranging from 79 to $94 \%$, although this dropped to $52 \%$ for the evaluation of small tumors. The size of the primary tumor was evaluated correctly in 32 of the 53 cases that underwent CT, resulting in a $60.3 \%$ accuracy rate. These results demonstrate the importance of acquiring as much information on the cancer as possible prior to surgery. Preoperative information acquired did not only contribute to the choice of the surgical intervention.

Neoadjuvant therapy was introduced in 2010, only 10 out of the 45 patients in the pre-PDTA group received this therapy even though at least 18 of them could have been included in stages of a neoadjuvant treatment. In contrast, for the patients operated on after January 2012, 31 of the 38 who should have received the neoadjuvant treatment ${ }^{1}$.

Similarly, patients affected by non-small cell lung cancer (NSCLC) require multimodal treatment with a combination of surgery, systemic chemotherapy and radiotherapy. This approach can have a palliative or curative intent and can involve multiple physicians and other healthcare professionals over the treatment period.

A recent retrospective single-centre analysis was performed by Tamburini et al. analyzed on consecutive patients who underwent surgery for non-small cell lung cancer (NSCLC) between January 2008 and December 2015 . A reduction in perioperative morbidity and mortality and improved survival in cohorts using a multidisciplinary team approach have been found.

The importance of the role of the multidisciplinary therapeutic diagnostic pathway in patient care and improvement of outcomes was sustained.

In this study, a total of 246 patients were treated prior to the initiation of the MTB and 231 patients after the initiation of the MTB. Based on the propensity score, 2 well-matched groups of 170 patients were identified.

Patients who were discussed at the MTB were noted to have better outcomes when compared with those who were not discussed at the MTB on different terms including complete staging evaluation, early tumour, node and metastasis (TNM) stages.

This study supports the view that multiprofessional lung cancer management improves the quality of care received by patients with NSCLC and the 1-year survival rate (odds ratio $0.48 ; 95 \%$ confidence interval $0.25-0.92)^{2}$.

Survival benefit was probably due to both the reduction in the number of advanced stage cancer patients proceeding to surgery, a more accurate selection of patients and the increased cumulative experience of the different specialists. These findings have led to recommend the management of all cancer patients in an MTB at our institution, irrespective of staging?

\section{Conclusion}

Although some available data are suggesting that managing lung cancer patients through MTB results in timely access to treatment and adherence to guidelines, to date, specific evidence is not available regarding the impact of this model of lung cancer care on survival or patient satisfaction. 
Because of the limited cases in the literature, it is not possible to draw definitive conclusions on this topic. It is desirable that new research is proposed in this area, also addressing lung cancer diseases as already performed in our institute.

\section{References}

1. Anania G, Resta G, Marino S, et al. Treatment of colorectal treatment: a multidisciplinary approach. J Gastrointest Cancer. 2018 Apr 14; 19416628 Online ISSN 1941-6636.

2. Tamburini N, Maniscalco P, Mazzara S, et al. Multidisciplinary management improves survival at 1 year after surgical treatment for non-small-cell lung cancer: a propensity score-matched study. Eur J Cardiothorac Surg. 2017 Dec 25; 53 (2018): 1199-1204 